\title{
A NOTE ON REGULAR RINGS WITH STABLE RANGE ONE
}

\author{
H. V. CHEN and A. Y. M. CHIN \\ Received 10 September 2001
}

\begin{abstract}
It is known that a regular ring has stable range one if and only if it is unit regular. The
\end{abstract} purpose of this note is to give an independent and more elementary proof of this result.

2000 Mathematics Subject Classification: 16E50, 16E65.

1. Introduction. All rings considered in this note are associative with identity. A ring $R$ is said to be (von Neumann) regular if, given any $x \in R$, there exists $y \in R$ such that $x y x=x$. If, given any $x \in R$, there exists an invertible element $u \in R$ such that $x u x=x$, then $R$ is said to be unit regular. A ring $R$ is said to have stable range one if for any $a, b \in R$ satisfying $a R+b R=R$, there exists $y \in R$ such that $a+b y$ is right invertible. By Vaserstein [4, Theorem 1], this definition is left-right symmetric.

It has been shown independently in $[1,3]$ that a regular ring has stable range one if and only if it is unit regular (see also [2]). The aim of this note is to provide a rather straightforward and more elementary proof of this result.

We need the following proposition.

Proposition 1.1. A ring $R$ has stable range one if and only if for any $a, x, b \in R$ satisfying $a x+b=1$, there exists $y \in R$ such that $a+b y$ is invertible.

Proof. Assume that $R$ has stable range one and let $a, x, b \in R$ satisfy $a x+b=1$. Then $a R+b R=R$ and by definition, there exists $y \in R$ such that $a+b y$ is right invertible. By [5, Theorem 2.6], it follows that $a+b y$ is left invertible. The converse is obvious.

We also need the following known result (see, e.g., [6]).

Proposition 1.2. Let $R$ be a ring. Then $R$ is unit regular if and only if every element of $R$ is the product of an idempotent and an invertible element (which do not necessarily commute).

2. A different proof. We are now ready to give a different proof of the following result.

THEOREM 2.1. A regular ring $R$ has stable range one if and only if it is unit regular.

Proof. First, assume that $R$ has stable range one and let $a \in R$. Since $R$ is regular, there exists $x \in R$ such that $a x a=a$. Clearly, $a x+(1-a x)=1$. By the assumption on $R$ and Proposition 1.1, there exists $y \in R$ such that $u=a+(1-a x) y$ is invertible. Therefore, $a \times u=a x[a+(1-a x) y]=a \times a=a$. It follows that $a x=a u^{-1}$ from which we have $a u^{-1} a=a x a=a$. 
Conversely, assume that $R$ is unit regular and suppose that $a x+b=1$ for some $a, x, b \in R$. By Proposition 1.2, we may write $a=e u, b=g v$ for some idempotents $e, g \in R$ and some invertible elements $u, v \in R$. It follows that

$$
e(u x+b)+(1-e) g v=e u x+e b+(1-e) b=a x+b=1 .
$$

Since $R$ is regular, there exists $c \in R$ such that $(1-e) g=(1-e) g c(1-e) g$. Let $f=$ $(1-e) g c(1-e)$. We then have, by $(2.1)$, that

$$
\begin{aligned}
e(u x+b)+f b & =e(u x+b)+(1-e) g c(1-e) g v \\
& =1-(1-e) g v+(1-e) g v=1 .
\end{aligned}
$$

Note that $0=f e u x=f a x=f(1-b)$, that is, $f b=f$. We also have $e=e 1=e(a x+$ $b)=e(u x+b)$. Thus

$$
e+f=e(u x+b)+f b=1 .
$$

It is clear that $1+e b v^{-1} c(1-e)$ is invertible with inverse $1-e b v^{-1} c(1-e)$. Since $e+f=1$, we have that $e+(1-e) g c(1-e)=1$, that is, $e+(1-e) g v v^{-1} c(1-e)=1$. But since $b=g v$, it follows that $e+(1-e) b v^{-1} c(1-e)=1$ and therefore

$$
e+b v^{-1} c(1-e)=1+e b v^{-1} c(1-e) \text {. }
$$

Since $(1-e) e=0$, we can write

$$
e+b v^{-1} c(1-e)\left[1+e b v^{-1} c(1-e)\right]=1+e b v^{-1} c(1-e) .
$$

Multiplying on the right by $u$ and noting that $e u=a$, we then obtain

$$
a+b v^{-1} c(1-e)\left[1+e b v^{-1}(1-e)\right] u=\left[1+e b v^{-1} c(1-e)\right] u,
$$

which is invertible. It then follows from Proposition 1.1 that $R$ has stable range one.

\section{REFERENCES}

[1] L. Fuchs, On a substitution property of modules, Monatsh. Math. 75 (1971), 198-204.

[2] K. R. Goodearl, von Neumann Regular Rings, 2nd ed., Robert E. Krieger Publishing, Florida, 1991.

[3] I. Kaplansky, Bass's first stable range condition, mimeographed notes, 1971.

[4] L. N. Vaserstein, Stable rank of rings and dimensionality of topological spaces, Funct. Anal. Appl. 5 (1971), 102-110.

[5] _ _ Bass's first stable range condition, J. Pure Appl. Algebra 34 (1984), no. 2-3, 319330.

[6] R. Yue Chi Ming, Remarks on strongly regular rings, Portugal. Math. 44 (1987), no. 1, 101112.

H. V. Chen: Institute of Mathematical Sciences, Faculty of SCIEnCE, University of MALAYA, 50603 KUALA LUMPUR, MALAYSia

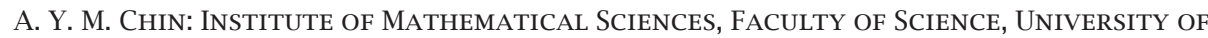
MALAYA, 50603 KuALa LUMPUR, MALAYSiA

E-mail address: acym@mnt. math.um. edu . my 


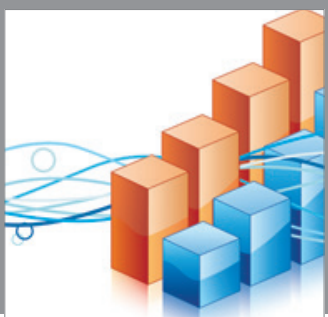

Advances in

Operations Research

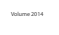

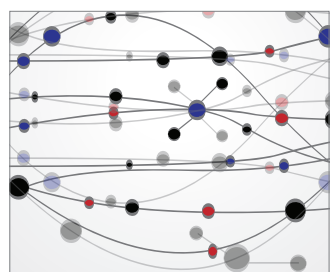

\section{The Scientific} World Journal
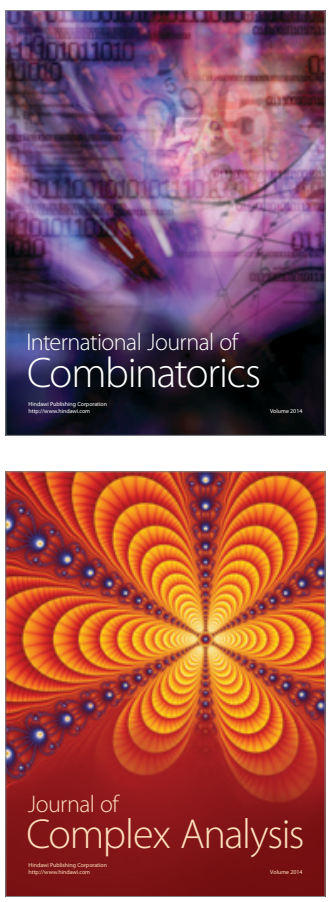

International Journal of

Mathematics and

Mathematical

Sciences
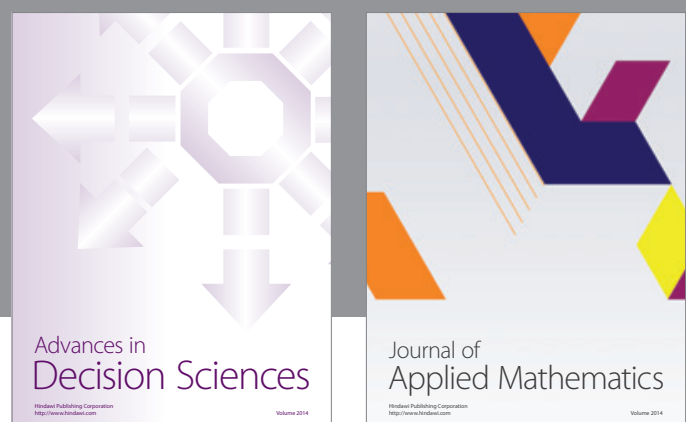

Journal of

Applied Mathematics
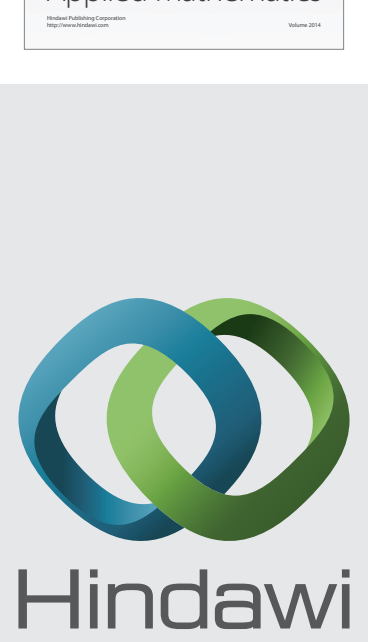

Submit your manuscripts at http://www.hindawi.com
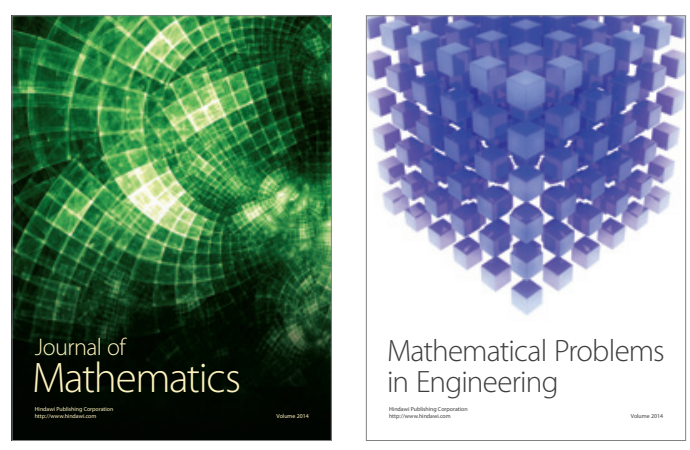

Mathematical Problems in Engineering
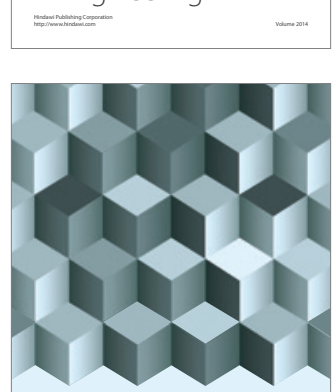

Journal of

Function Spaces
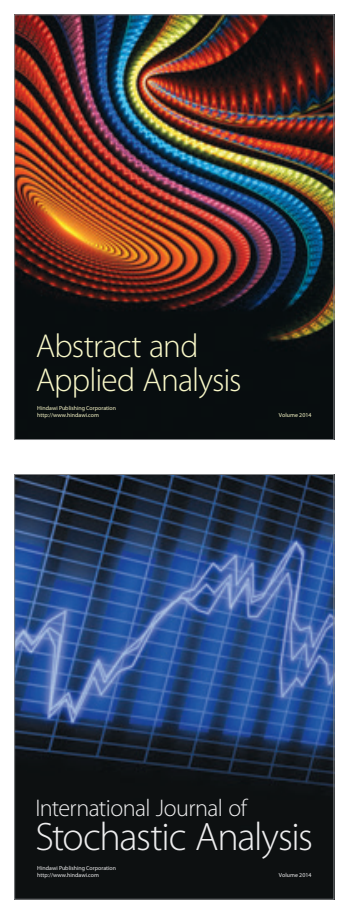

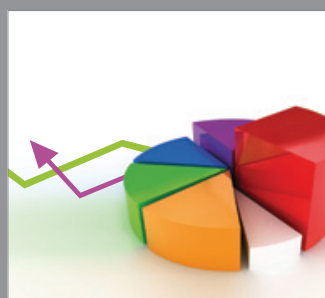

ournal of

Probability and Statistics

Promensencen
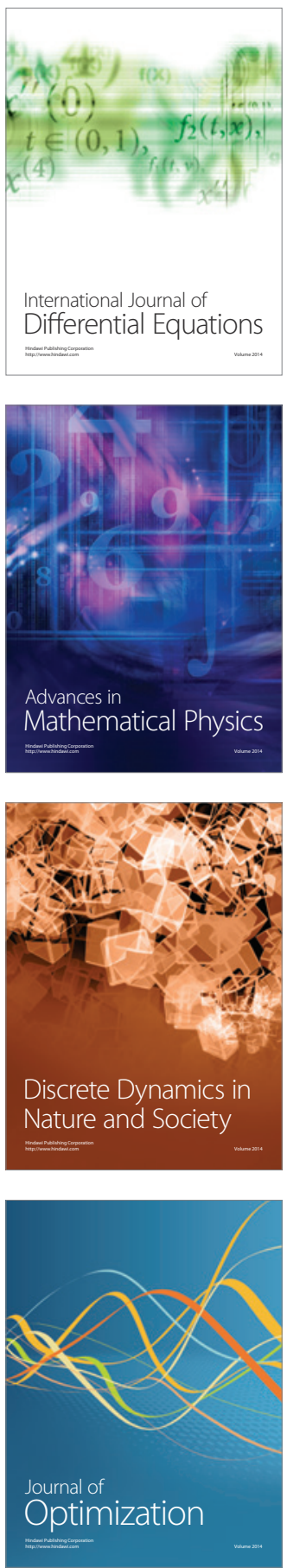